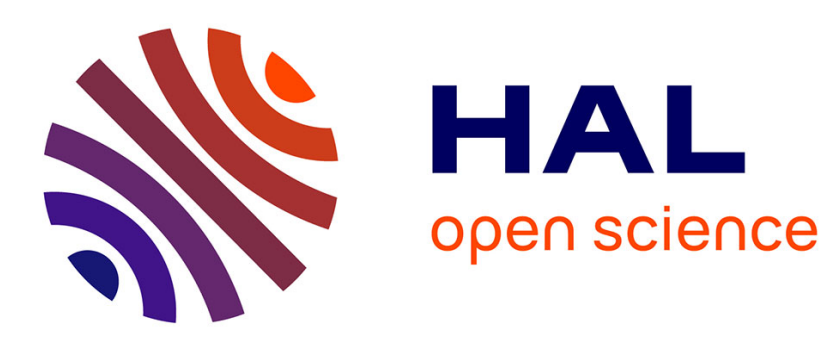

\title{
Étude des changements morphologiques d'une rivière en tresses par LiDAR aéroporté
}

\author{
S. Lallias-Tacon, F. Liebault, H. Piégay
}

\section{To cite this version:}

S. Lallias-Tacon, F. Liebault, H. Piégay. Étude des changements morphologiques d'une rivière en tresses par LiDAR aéroporté. IS Rivers 2012, Jun 2012, Lyon, France. 3 p. hal-00967391

\section{HAL Id: hal-00967391 \\ https://hal.science/hal-00967391}

Submitted on 28 Mar 2014

HAL is a multi-disciplinary open access archive for the deposit and dissemination of scientific research documents, whether they are published or not. The documents may come from teaching and research institutions in France or abroad, or from public or private research centers.
L'archive ouverte pluridisciplinaire HAL, est destinée au dépôt et à la diffusion de documents scientifiques de niveau recherche, publiés ou non, émanant des établissements d'enseignement et de recherche français ou étrangers, des laboratoires publics ou privés. 


\title{
Étude des changements morphologiques d'une rivière en tresses par LiDAR aéroporté

\author{
LiDAR-derived moprhological changes of a braided river \\ in the French Prealps
}

\author{
Sandrine Lallias-Tacon ${ }^{1,2}$, Frédéric Liébault ${ }^{1}$ and Hervé Piégay ${ }^{2}$ \\ ${ }^{1}$ Irstea Grenoble, Unité de Recherche ETNA, Domaine universitaire, 2 rue de la \\ Papeterie, BP76, 38402 Saint-Martin-d'Hères (corresponding author: \\ sandrine.tacon@irstea.fr). ${ }^{2}$ Université de Lyon, CNRS-UMR5600, Site Ens \\ Lyon, 15 Parvis R. Descartes, F-69342 Lyon. \\ Zone Atelier Bassin du Rhône
}

\section{RÉSUMÉ}

Des levés LiDAR aéroportés multi-temporels sont utilisés pour reconstruire les changements morphologiques d'un lit en tresses suite à une crue morphogène. Ces levés ont été acquis en octobre 2008 et avril 2010 sur un linéaire de 7 km du Bès, un affluent de la Bléone dans les Préalpes du Sud. Au cours de cette période, une crue de récurrence 15 ans a été enregistrée en décembre 2009. Les deux MNT ont été soustraits pour obtenir une carte d'érosion et de dépôt. Des mesures topographiques au dGPS ont été réalisées sur des surfaces aux caractéristiques différentes pour évaluer la variabilité des erreurs dans les MNT. La carte d'érosion et de dépôt permet d'étudier les changements morphologiques à l'échelle des macroformes et de calculer un bilan sédimentaire. Ces changements morphologiques sont enfin replacés dans leur contexte historique, étudié à partir des campagnes de photographies aériennes (1948-2010) pour lier la morphologie du lit actuel avec les changements morphologiques à long terme.

\begin{abstract}
Multidate airborne LiDAR surveys were used to reconstruct reach-scale morphological changes of a gravel-bed braided river following a channel-forming flood event. LiDAR surveys were acquired in October 2008 and June 2010 for a 7-km reach of the Bès River, a very active aggrading braided channel, which is a tributary to the Bléone River in the Southern French Prealps. Between these two dates, a 15-year flood occurred in December 2009. A DEM of difference (DoD) was produced by subtraction of LiDAR-derived DTM pair. Spatially distributed error in Dod was accounted with dGPS field measurement by sampling of different types of terrains (exposed gravel bars, spares and dense vegetated areas). The scour and fill maps allowed reconstructing erosion and deposition of bedforms and provide a volume estimate for calculating a sediment budget. These morphological changes were also studied in a historical context with a serie of aerial photographs (1948-2010) to link the presentday morphology of the river with longer term channel changes.
\end{abstract}

\section{MOTS CLES}

Airborne LiDAR, braided river, flood, uncertainty error assessment. 


\section{INTRODUCTION}

L'utilisation du LiDAR pour la caractérisation des formes fluviales s'est développée très rapidement au cours des dix dernières années, ce qui a permis des avancés importantes en matière de reconstitution de la morphodynamique des lits fluviaux. Ces nouvelles technologies permettent l'acquisition rapide de données topographiques à de fines résolutions spatiales et sur de grandes étendues spatiales. Ces données permettent de réaliser facilement des Modèles Numériques de Terrain (MNT) de haute résolution. La réalisation de levés topographiques répétés permet de suivre les changements morphologiques et d'estimer le budget sédimentaire.

Dans cette étude, des levés LiDAR aéroportés multi-temporels sont utilisés pour reconstruire les changements morphologiques d'une rivière en tresses liés à une crue morphogène.

\section{PRESENTATION DU SITE D'ETUDE}

\subsection{Le bassin versant du Bès}

La rivière du Bès est un affluent de rive droite de la Bléone. Son bassin versant de $234 \mathrm{~km}^{2}$ se situe au NNE de Digne-les-Bains. Le linéaire d'étude intègre les 7 derniers kilomètres du Bès, caractérisés par un tressage très actif, en exhaussement à l'échelle du $20^{\mathrm{e}}$ siècle. La pente du lit est de $1,4 \%$. La largeur moyenne de bande active est de $220 \mathrm{~m}$, variant de 50 à $230 \mathrm{~m}$. Une station hydrologique de la DREAL est située en amont du secteur d'étude, sur la commune d'Escanglon-Péroure. Les données hydrologiques sont disponibles depuis 1965. Le régime hydrologique est nivo-pluvial avec des hauteseaux printanières dues à la fonte des neiges, un étiage estival et des hautes eaux automnales dues à des précipitations intenses.

\subsection{Caractéristique hydrologique de la période d'étude}

Entre les levés LiDAR du 27/10/2008 et du 19/04/2010, le Bès a subi deux pics de crue successifs les 22 et 24 décembre 2009, de période de retour 15 et 20 ans respectivement, avec un débit maximum instantané de $171 \mathrm{~m}^{3} / \mathrm{s}$. Sur le reste de l'intervalle de temps entre les deux levés LIDAR, les débits sont restés faibles, avec des hautes eaux printanières d'intensité modérée. Les changements morphologiques observés sur cette période sont donc la conséquence principale de la crue de décembre 2009.

\section{METHODOLOGIE}

Des levés LiDAR aéroportés ont été réalisés en octobre 2008 et en avril 2010 par la société Sintégra. Le nuage de points bruts a été classé en "points sol" et "sursol" avec le filtre automatique d'Axellson du logiciel TerraScan. Les MNT ont été construits dans ArcGIS en créant des TIN selon la triangulation de Delauney à partir du nuage de "points sol", puis des points sont échantillonnés linéairement sur la facette des triangles en une grille $\mathrm{d}^{\prime} 1 \mathrm{~m}^{2}$.

La soustraction du MNT de 2010 avec celui de 2008 sur une base de cellule par cellule $\left(1 \mathrm{~m}^{2}\right)$ permet de calculer la différence d'altitude entre les deux dates. Les volumes érodés et déposés peuvent ainsi être estimés pour calculer le bilan sédimentaire. Lors de cette étape, une estimation de l'erreur d'incertitude des changements d'altitude observés est nécessaire. Un grand nombre de facteurs peuvent introduire des erreurs dans les MNT incluant la qualité du levé des points, la stratégie d'échantillonnage, la composition de la surface, la complexité de la topographie et les méthodes d'interpolations (Milan et al., 2011). Des altitudes mesurées au dGPS sur des surfaces aux caractéristiques différentes (granulométrie et degré de végétalisation variables) ont été comparées aux altitudes du MNT pour estimer la variabilité dans l'erreur d'incertitude selon la complexité du terrain.

Afin de replacer ces changements morphologiques à court terme avec la trajectoire à long terme du Bès, une étude diachronique de l'évolution de la bande active du Bès a été réalisée à partir de campagnes de photographies aériennes de 1948 à 2010. Les photographies aériennes ont été géoréférencées avec des points de calage à partir des orthophotographies de 2004. La bande active, qui correspond aux surfaces en eau et aux bancs non végétalisés, a été digitalisée pour chaque date et sa largeur a été mesurée tous les $10 \mathrm{~m}$ suivant l'axe longitudinal de la rivière. 


\section{RESULTATS ET DISCUSSION}

Différence d'altitude (en $\mathrm{m}$ )

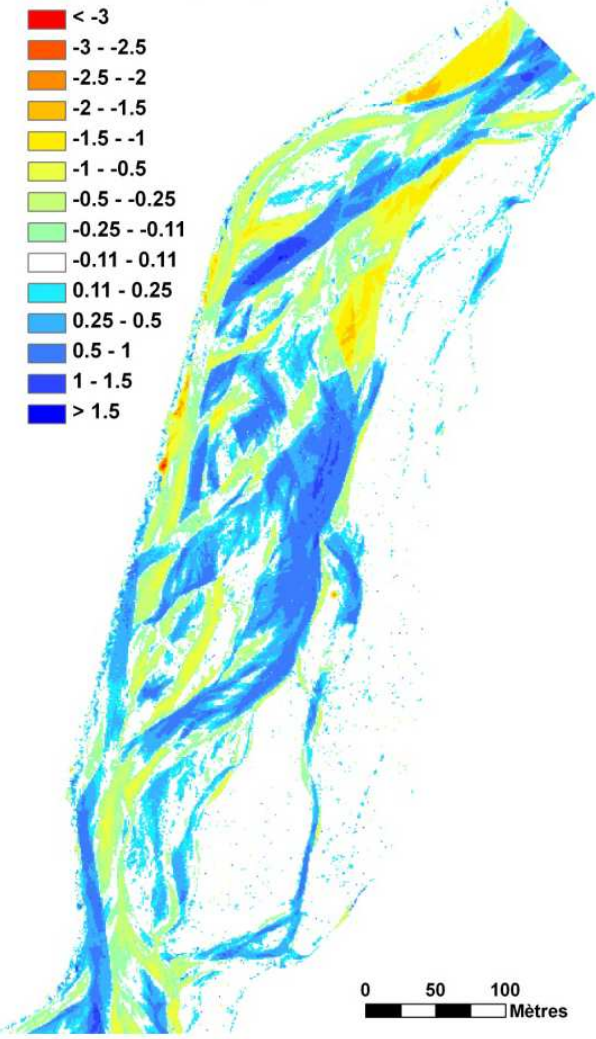

Figure 1 : MNT de la différence d'altitude du lit du Bès entre 2008 et 2010 sur le secteur amont (avec une limite de détection de $0,118 \mathrm{~m}$ ).

L'étude diachronique des photographies aériennes a mis en évidence une légère diminution de la largeur moyenne de la bande active entre 1948 et 2010. Le Bès conserve donc une importante bande de tressage active.

Ces résultats confirment que le Bès est une des dernières rivières des Alpes françaises ayant un tressage encore actif et un bilan sédimentaire positif. En effet, ce style fluvial est devenu peu fréquent en raison d'une réduction régionale des sédiments délivrés (Liébault et al., 2010). La compréhension du fonctionnement morphologique de ces systèmes est donc essentielle pour leur préservation.
Le Bès a subi des changements morphologiques significatifs pendant la crue de décembre 2009 (Figure 1). Des processus morphologiques sont clairement observables comme des érosions de berge, des dépôts, et des migrations de chenal principal.

Des premières mesures topographiques au dGPS ont permis de calculer une limite de détection uniforme des changements morphologiques de $0.118 \mathrm{~m}$, d'après la formule de propagation des erreurs dans la soustraction de deux MNT (Brasington et al., 2003). Avec cette limite de détection, le bilan sédimentaire s'élève à $+47091 \mathrm{~m}^{3}$. Des mesures topographiques au dGPS sur des surfaces aux caractéristiques différentes ont permis de préciser cette erreur d'incertitude. Les résultats ont montré que les altitudes dérivées du LiDAR de 2010 surestiment systématiquement les altitudes mesurés au dGPS d'environ $6 \mathrm{~cm}$ et que plus les surfaces se complexifient (augmentation du degré de végétalisation) plus l'erreur d'incertitude augmente. La prise en compte de ces différences d'incertitudes dans le bilan sédimentaire permettra d'améliorer le calcul du bilan sédimentaire et d'affiner la compréhension de certains processus morphologiques.

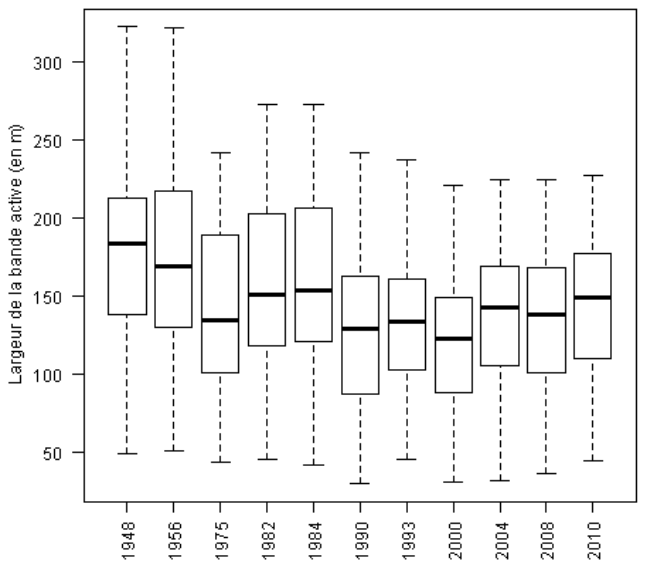

Figure 2 : Evolution de la bande active du Bès entre 1948 et 2010.

\section{BIBLIOGRAPHIE}

Brasington J, Langham J, Rumsby B. (2003). Methodological sensitivity of morphometric estimates of coarse fluvial sediment transport. Geomorphology 53, 299-316.

Liébault F., Cassel M., Jantzi H., Tacon S., Talaska N. (2010). Régime sédimentaire et morphohologie des rivières en tresses alpines et méditerrannéennes. Cemagref, 28p.

Milan DJ, Heritage GL, Large ARG, Fuller IC. (2011). Filtering spatial error from DEMs: Implications for morphological change estimation. Geomorphology 125, 160-171. 